\title{
Acumulação de massa seca e rendimento de frutos de tomateiro cultiva- do em substrato com cinco doses de solução nutritiva
}

\author{
Jorge E. Rattin ${ }^{1}$; Jerônimo L. Andriolo ${ }^{2}$; Márcio Witter ${ }^{2}$ \\ ${ }^{1}$ Facultad de Ciências Agrárias, Universidad Nacional de Mar del Plata. RN 226, km 73,5. Balcarce, Pcia. de Buenos Aires, Argentina; \\ ${ }^{2}$ UFSM-CCR-Depto. de Fitotecnia, 97.105-900, Santa Maria-RS. E-mail: andriolo@creta.ccr.ufsm.br.
}

\section{RESUMO}

Avaliou-se o efeito de cinco doses de solução nutritiva sobre a acumulação de massa seca e rendimento de frutos do tomateiro, híbrido Monte Carlo, cultivado em substrato no interior de uma estufa de polietileno. A semeadura foi feita em 09/07/99 e 32 dias após as mudas foram transferidas para sacolas de polietileno com 4,5 $\mathrm{L}$ de substrato comercial, na densidade de 3,3 plantas $/ \mathrm{m}^{2}$. Foi empregada como referência uma solução nutritiva contendo, em mmol/L: $40 \mathrm{de}$ $\mathrm{KNO}_{3} ; 27 \mathrm{de} \mathrm{Ca}\left(\mathrm{NO}_{3}\right)_{2} ; 12$ de $\mathrm{MgSO}_{4}$, complementada por $1,5 \mathrm{~g} / \mathrm{L}$ de superfosfato simples, $0,13 \mathrm{ml} / \mathrm{L}$ de Fe quelatizado e $0,66 \mathrm{ml} / \mathrm{L}$ de uma solução de micronutrientes. $\mathrm{O}$ tratamento $\mathrm{T} 3$ foi igual à dose de referência e os demais tratamentos foram fixados em doses múltiplas de T3, multiplicando-se as quantidades de todos os nutrientes por 0,$25 ; 0,50 ; 1,25$ e 1,50, para os tratamentos T1, T2, T4 e T5, respectivamente. Em cada tratamento, o volume de $1 \mathrm{~L}$ de solução foi aplicado para cada planta em intervalos semanais, por fertirrigação. Determinou-se o acúmulo de massa seca aos 138 dias após a semeadura e a produtividade de frutos maduros ao final do ciclo da cultura. A resposta da acumulação de massa seca total da parte aérea e de frutos se ajustou a uma equação linear, enquanto a massa seca vegetativa, o índice de área foliar e a produtividade se ajustaram a um modelo quadrático. A concentração de 101,5 mmolN/ L, com a devida proporção entre os demais nutrientes, foi estimada como aquela a ser empregada para atingir a produtividade máxima de frutos dessa cultura cultivada em substratos.

Palavras-chave: Lycopersicon esculentum, fertirrigação, estufa, cultivo fora do solo.

\begin{abstract}
Dry mass accumulation and fruit yield of tomato plants grown in substrate under five doses of nutrient solution
\end{abstract}

We evaluated the effect of five doses of nutrient solution on dry matter accumulation and fruit yield of tomato plants, hybrid Monte Carlo, grown in substrate inside a polyethylene greenhouse. Sowing was done on August $9^{\text {th }}, 1999$, and 32 days later plants were transferred to bags with $4.5 \mathrm{~L}$ of a commercial substrate, at a plant density of 3.3 plants $/ \mathrm{m}^{2}$.As reference we used a nutrient solution containing, in mmol/L: $\mathrm{KNO}_{3}, 40 ; \mathrm{Ca}\left(\mathrm{NO}_{3}\right)_{2}, 27 ; \mathrm{MgSO}_{4}, 12$, complemented by $1.5 \mathrm{~g} / \mathrm{L}$ of superphosphate $\left(20 \% \mathrm{P}_{2} \mathrm{O}_{5}\right), 0.13 \mathrm{ml} / \mathrm{L}$ of Fe-chelate and $0.66 \mathrm{ml} / \mathrm{L}$ of a mixture of micronutrients. The T3 treatment was equal to the reference nutrient solution, whereas in treatments T1, T2, T4 and T5 quantities of all nutrients from T3 were multiplied by $0.25 ; 0.50 ; 1.25$ e 1.50 , respectively. In each treatment, the volume of $1 \mathrm{~L}$ of nutrient solution was supplied to each plant once a week by fertigation. Plant dry mass accumulation was determined at 138 days after sowing, and fruit yield at the end of the growing period. Data from total shoot dry mass fitted well on a linear regression, whereas from vegetative dry mass, leaf area index and fruit yield a quadratic model was adjusted. The concentration of $101.5 \mathrm{mmolN} / \mathrm{L}$, with the proportion among other nutrients of the nutrient solution being respected, was considered as the one to be used to reach the maximum fruit yield of this crop grown in substrates.

Keywords: Lycopersicon esculentum, fertigation, greenhouse, soilless culture.

(Recebido para publicação em 15 de maio de 2001 e aceito em 13 de setembro de 2002)

$\mathrm{O}$ cultivo do tomateiro em ambiente protegido é uma das técnicas empregadas para obter elevadas produtividades nos períodos de entressafra, superiores a $100 \mathrm{t} / \mathrm{ha}$ em ciclo de aproximadamente 150 dias (Andriolo et al., 1997). Para atingir tais rendimentos, são necessárias doses adequadas de nutrientes, normalmente elevadas. Inúmeras recomendações de adubação para uso regional encontram-se na literatura, com forte discrepância entre os valores. Essas diferenças provém tanto do fato de que o potencial de produção é variável entre as diferentes cultivares e híbridos empregados, como das reações no meio radicular que variam quando os cultivos são efetuados em diferentes condições, tanto no solo como em substrato (Van
Keulen \& Stol, 1991; Van Raij, 1991; Fayad, 1998). Conseqüentemente, problemas nutricionais são freqüentes.

No estado do Rio Grande do Sul, Veduim \& Bartz (1998) observaram aumento da concentração de nutrientes no solo de 44 estufas localizadas em distintas regiões. $\mathrm{O}$ excesso de nutrientes minerais pode conduzir à salinização e/ou antagonismo entre os nutrientes, com efeitos negativos sobre a produção. Uma das alternativas para facilitar o manejo da nutrição mineral das hortaliças consiste em empregar o cultivo em substratos com o uso da fertirrigação. Por meio dessa técnica é possível proteger as plantas tanto dos desequilíbrios nutricionais como das pragas e moléstias do solo (CTIFL, 1995; Cortés, 1999). Outra vantagem é o aumen- to da eficiência de uso da água, pois as perdas por evaporação superficial são reduzidas (Papadoupoulos, 1997).

A maior parte dos cultivos de tomateiro em ambiente protegido no hemisfério Norte é realizada em substrato inerte, empregando solução nutritiva completa, que é fornecida diurnamente de forma a suprir a demanda hídrica da cultura (Le Bot et al., 1998; Cortés, 1999; CTIFL, 1995). Andriolo \& Poerschke (1997) utilizaram uma dose semanal de nutrientes fornecida por fertirrigação, sendo essa dose estimada a partir da quantidade de nutrientes fornecida às plantas cultivadas pelo método clássico empregado naqueles países, descontando-se a fração perdida pela drenagem (Andriolo et al., 1997). 
No Brasil, são escassos os estudos feitos com o objetivo de estabelecer critérios técnicos para o manejo da adubação na produção comercial do tomateiro, tais como a dose econômica, frequiência e modo de aplicação (Takahasi, 1993; Fontes \& Guimarães, 1999). Em Santa Maria, Grave (1999) determinou o efeito de diferentes doses de $\mathrm{N}$ sobre a acumulação e distribuição da massa seca entre os órgãos da parte aérea do tomateiro, híbridos Diva e Monte Carlo, ambos de crescimento indeterminado, cultivados em substrato orgânico. As doses comparadas com o híbrido Diva foram de 1,3; 1,5 e $1,7 \mathrm{~g}$ de $\mathrm{N}$ por planta e com o híbrido Monte Carlo de 0,75; 1,5 e 2,34 g de $\mathrm{N}$ por planta, aplicadas em intervalos semanais. Os demais nutrientes foram mantidos constantes, de acordo com as quantidades recomendadas por Andriolo \& Poerschke (1997). Em ambos os experimentos, não foram constatadas diferenças significativas entre os tratamentos. Fayad (1998) determinou a extração de macro e micronutrientes para os híbridos EF-50 e Santa Clara, tanto em cultivo no campo como em estufa. Entretanto, critérios de manejo capazes de indicar a dose de nutrientes a fornecer quando as plantas são cultivadas em substrato não foram apontados neste trabalho. Em outros países, tem sido demonstrado que a disponibilidade de nutrientes no meio radicular dessa espécie em cultivo fora do solo afeta a acumulação e repartição da massa seca, o rendimento e também a qualidade dos frutos (Ehret \& Ho, 1986; Huett , 1989; Larouche et al., 1989; Adams et al., 1994; CTIFL, 1995; Cortés, 1999).

O presente trabalho teve como objetivo determinar o efeito de cinco doses de solução nutritiva sobre a acumulação de massa seca e o rendimento de frutos de tomateiro cultivado em substrato e com fertirrigação.

\section{MATERIAL E MÉTODOS}

$\mathrm{O}$ experimento foi instalado no Depto. de fitotecnia da UFSM (RS), (latitude $29^{\circ} 43^{\prime} \mathrm{S}$, longitude $53^{\circ} 42 \mathrm{~W}$ e altitude $95 \mathrm{~m}$ ), numa estufa de polietileno de $200 \mathrm{~m}^{2}$. O híbrido Monte Carlo, de crescimento indeterminado, foi semeado no dia 9 de julho de 1999 , em bandejas de poliestireno contendo substrato comercial. Aos 32 dias após a semeadura (DAS), as mudas com quatro folhas definitivas foram transplantadas em sacolas de polietileno contendo 4,5 L de substrato orgânico (Plantmax $\left.{ }^{\circledR}\right)$, localizadas no interior da estufa. Sobre as sacolas foi instalado um tubo gotejador para o fornecimento de água e nutrientes, ajustando-se um gotejador no centro de cada sacola. $\mathrm{O}$ conjunto formado pelas sacolas e o tubo gotejador foi coberto com filme de polietileno opaco de cor preta, para reduzir a evaporação superficial e evitar a incidência direta dos raios solares sobre as sacolas.

O substrato foi mantido com o teor de umidade sempre próximo da capacidade máxima de retenção. A irrigação foi suspensa imediatamente aos primeiros sinais de escorrimento, a fim de reduzir as perdas de nutrientes por lixiviação. Para permitir a livre drenagem da água excedente, as sacolas foram perfuradas na base e colocadas sobre camalhões orientados no sentido longitudinal da estufa.

As plantas foram dispostas em um arranjo de $1 \mathrm{~m}$ entre fileiras e $0,30 \mathrm{~m}$ entre plantas na mesma fileira, na densidade de 3,3 plantas $/ \mathrm{m}^{2}$, e conduzidas verticalmente com uma haste por meio de fitas plásticas. Uma vez por semana foi feita a poda das ramificações laterais. A estufa foi ventilada nos dias ensolarados, pela abertura das cortinas laterais e portões de acesso, entre as 9:00 h e 18:00 h, aproximadamente.

Do transplante até o início da floração, os nutrientes foram fornecidos às plantas uma vez por semana, via fertirrigação. A solução nutritiva empregada como referência foi aquela recomendada por Andriolo \& Poerscke (1997), para o cultivo do tomateiro em substrato, com a seguinte composição, em mmol/L: 40 de $\mathrm{KNO}_{3} ; 27$ de $\mathrm{Ca}\left(\mathrm{NO}_{3}\right)_{2} ; 12$ de $\mathrm{MgSO}_{4}$. O fósforo foi fornecido pela dissolução de $1,5 \mathrm{~g} / \mathrm{L}$ de superfosfato simples, eliminando-se as impurezas por decantação. O ferro foi fornecido na forma quelatizada (5\% de $\mathrm{Fe}$ ), na proporção de $0,13 \mathrm{ml} / \mathrm{L}$, e os demais micronutrientes através de 0,66 $\mathrm{ml} / \mathrm{L}$ da solução de micronutrientes pro- posta por Jeannequin (1987), descrita por Andriolo (1999). A proporção entre os macronutrientes na solução empregada foi de 1-0,23-1,18-0,82-0,23-0,3, respectivamente para o $\mathrm{N}-\mathrm{P}-\mathrm{K}-\mathrm{Ca}-\mathrm{Mg}-\mathrm{S}$. Foi fornecido o volume de $1 \mathrm{~L}$ por planta, em intervalos semanais, até o início da floração, totalizando $5 \mathrm{~L}$ por planta no período. A partir desse estádio, foram aplicadas semanalmente cinco doses de nutrientes, como tratamentos. O tratamento T3 foi igual à dose de referência, contento a concentração de nitrogênio de 94 $\mathrm{mmol} / \mathrm{L}$. Os demais tratamentos corresponderam às quantidades de nutrientes de T3 multiplicadas por 0,25 ; 0,$50 ; 1,25$ e 1,50 para os tratamentos $\mathrm{T} 1$, T2, T4 e T5, respectivamente. Mediuse diariamente a condutividade elétrica (CE) da solução drenada, em cada um dos tratamentos. Para tal, foi coletado separadamente o volume drenado de cinco plantas, no interior de uma parcela, em cada um dos tratamentos.

$\mathrm{O}$ delineamento experimental empregado foi o inteiramente casualizado, com três repetições. Cada uma das parcelas, formadas por 22 plantas, foi subdividida em dois grupos de onze plantas. No primeiro grupo, mediu-se a produção de frutos colhidos maduros. No segundo grupo, foi coletada ao acaso uma planta por parcela aos 138 DAS, para efetuar medidas destrutivas de análise de crescimento. As plantas coletadas foram separadas em caule (incluindo pecíolos e pedúnculos), folhas e frutos e submetidas à secagem em estufa, à temperatura de $60^{\circ} \mathrm{C}$, até peso constante. Os frutos maduros foram colhidos duas vezes por semana, no período entre 130 DAS e 156 DAS, ao apresentarem a coloração rosada ou vermelha, e pesados. A produtividade foi obtida pelo somatório da produção de todas as colheitas, em cada um dos tratamentos. $\mathrm{O}$ experimento foi encerrado no dia 12 de dezembro de 1999, aos 156 DAS.

\section{RESULTADOS E DISCUSSÃO}

Os valores médios semanais de $\mathrm{CE}$ da solução drenada confirmaram os cinco níveis nutricionais fornecidos pelas soluções nutritivas (Figura 1). Os valores dessa variável flutuaram em todos 


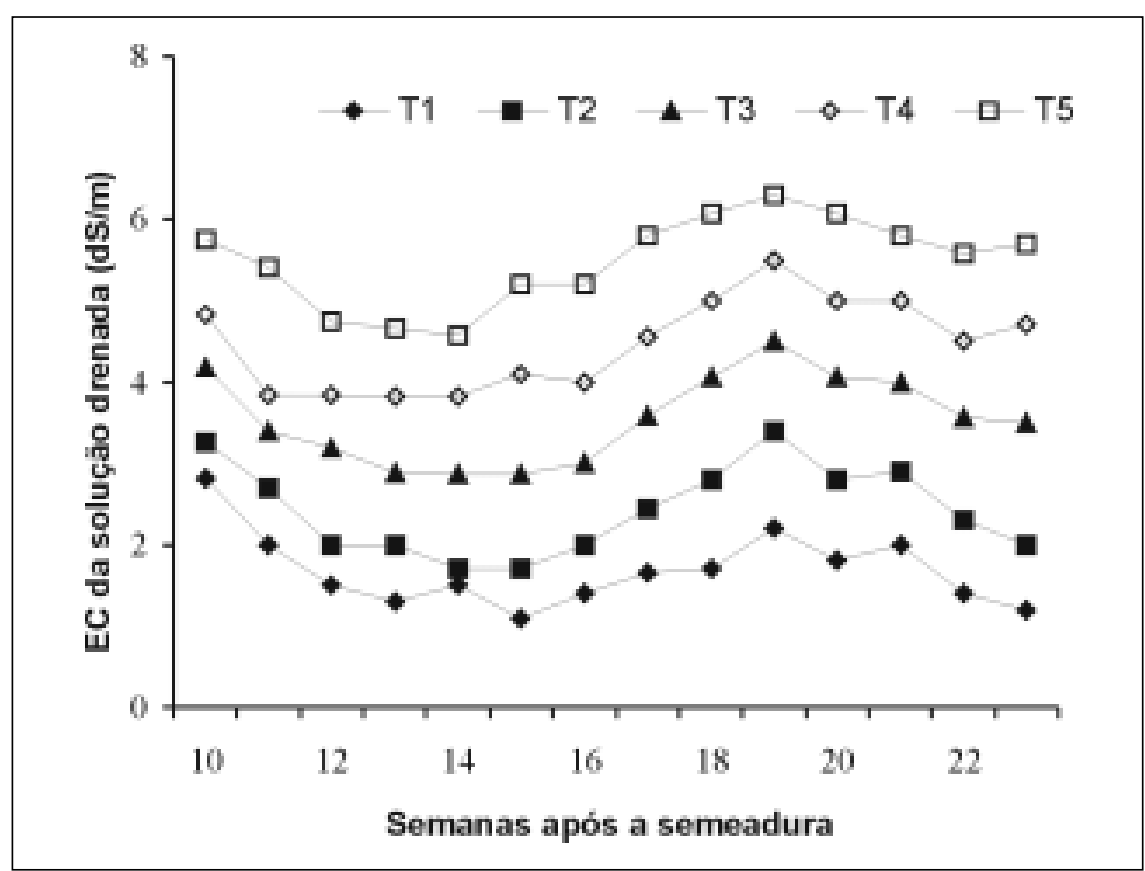

Figura 1. Evolução dos valores médios semanais de condutividade elétrica (CE) da solução drenada durante o experimento, para as cinco doses de solução nutritiva (T1 a T5). Santa Maria, UFSM, 2000
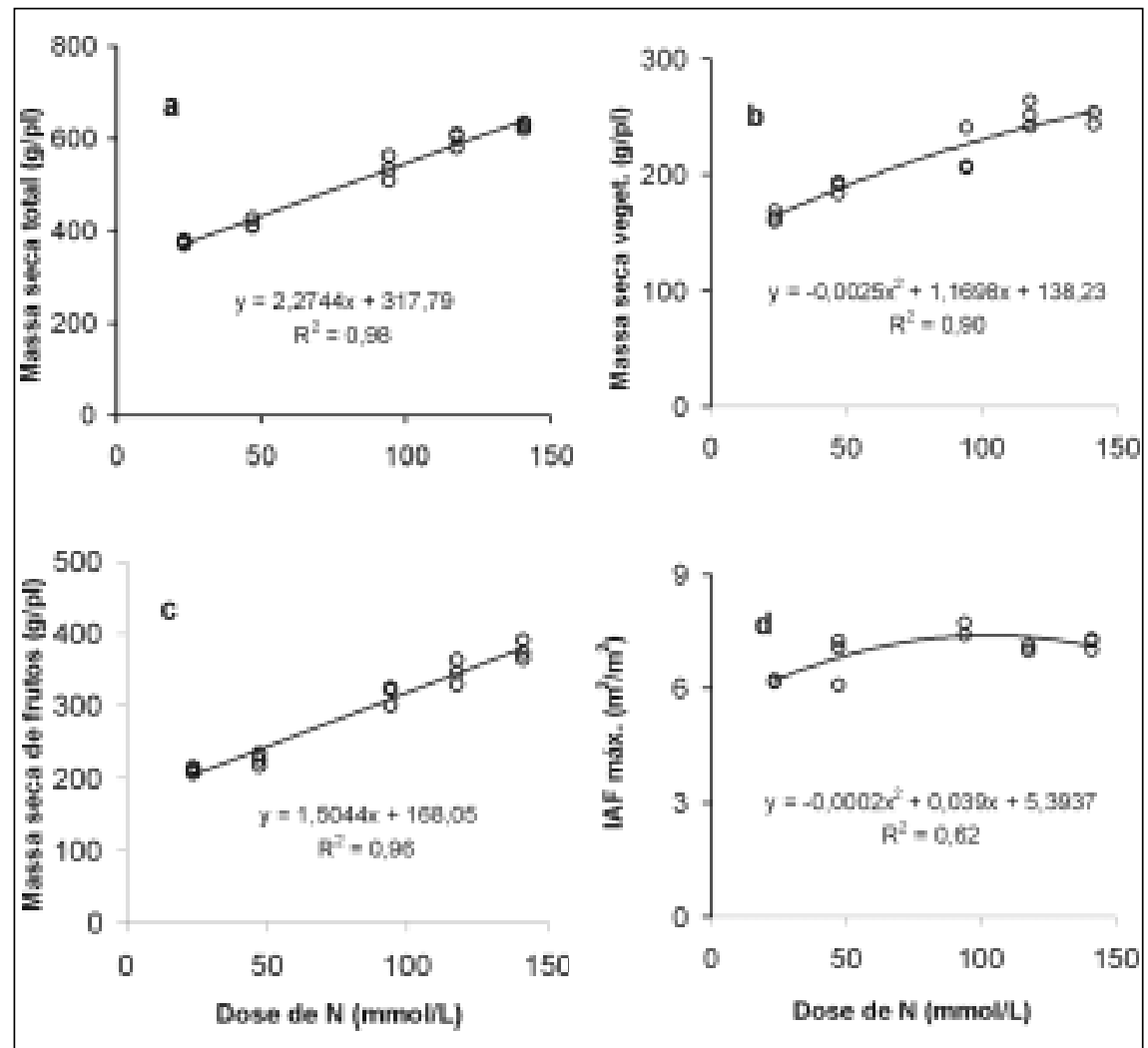

Figura 2. Evolução da acumulação de massa seca total da parte aérea (a), dos órgãos vegetativos (b), dos frutos (c) e do índice de área foliar (IAF) (D) do tomateiro, aos 138 dias após a semeadura, para as cinco doses de solução nutritiva, empregando-se o $\mathrm{N}$ como referência. (As doses foram identificadas pelo seu teor de N, de 23,5; 47,0; 94,0; 117,5 e 141,0 $\mathrm{mmol} / \mathrm{L}$, respectivamente para T1, T2, T3, T4 e T5, mantendo-se a proporção entre todos os nutrientes). Santa Maria, UFSM, 2000. os tratamentos, no decorrer do período experimental. Essa flutuação é atribuída às variações no fluxo de transpiração da planta, pois a CE da solução drenada aumenta à medida que diminui o teor de água no interior do substrato (Cortés, 1999). Os valores máximos medidos no tratamento T5, em torno de $6,0 \mathrm{dS} / \mathrm{m}$, situaram-se dentro do limite superior tolerado pela planta do tomateiro, próximo de 7,0 dS/m (Ehret \& Ho, 1986; Adams, 1994).

A produção de massa seca total medida aos 138 DAS mostrou resposta linear às doses de nutrientes (Figura 2a). $\mathrm{O}$ valores foram de $375 ; 415 ; 534 ; 599$ e $628 \mathrm{~g} /$ planta, para T1, T2, T3, T4 e $\mathrm{T} 5$, respectivamente. A massa seca dos órgãos vegetativos da parte aérea da planta apresentou resposta do tipo quadrático, com valores de 164; 190; $218 ; 252$ e $251 \mathrm{~g} / \mathrm{planta}$, respectivamente para os mesmos tratamentos (Figura 2b). A resposta da massa seca de frutos foi linear, com valores de 211; 226; 316; 347 e 381 g/planta (Figura 2c), enquanto aquela do IAF se ajustou a uma função quadrática, com valores de 6,$1 ; 6,8$; 7,5; 7,1 e 7,2 (Figura 2d), respectivamente, para os mesmos tratamentos. A fração da massa seca alocada para os frutos variou entre 0,54, em T2, e 0,61, em T5. A produtividade de frutos colhidos maduros ao final do experimento evidenciou resposta quadrática às doses de nutrientes (Figura 3 ) e os valores medidos foram de 1,9; 2,6; 3,3, 3,6 e $3,3 \mathrm{~kg} /$ planta, respectivamente para os cinco tratamentos.

Causa surpresa a resposta linear observada na acumulação de massa seca total e de frutos ao aumento das doses de nutrientes fornecidos, uma vez que a CE das soluções nutritivas recomendadas para o cultivo do tomateiro em substrato se situam entre 2,0 e 3,0 dS/m (CTIFL, 1995; Perez \& Lopez, 1998; Cortés, 1999), abaixo dos valores médios obtidos no atual experimento. Resultados recentes de Le Bot et al. (2001) sugerem que esses limites recomendados deveriam ser reduzidos, porque estariam acima das necessidades do tomateiro. Entretanto, os dados de literatura provenientes de países do Hemisfério Norte dizem respeito à fertirrigação contínua, onde uma dose de solução nutri- 
tiva completa com valor de CE dentro da faixa ótima é fornecida diuturnamente, sempre que necessária para repor o volume de água perdido pela transpiração. No caso da fertirrigação descontínua, como aquela empregada no atual experimento, os valores de CE decrescem entre duas fertirrigações sucessivas, o que implica que nos dias anteriores à fertirrigação seguinte a disponibilidade de nutrientes poderia estar limitando o crescimento das plantas. Uma das possibilidades para evitar essa situação, seria realizar a fertirrigação de acordo com a variação da $\mathrm{CE}$ da solução drenada. Essa variável seria monitorada diariamente e uma nova dose seria fornecida assim que os valores medidos de CE mostrassem decréscimo em relação à faixa ótima. Esse procedimento se aproximaria daquele praticado naqueles países. Entretanto, implicaria duas inconveniências: a primeira, de aumentar as quantidades de nutrientes perdidas pela drenagem, pois para evitar o aumento excessivo da CE nos períodos de forte transpiração, a freqüência das irrigações e a fração drenada teriam que ser aumentadas. A segunda, de aumentar o custo da fertirrigação, o que poderia inviabilizar a técnica no contexto econômico atual da produção de hortaliças. Outra possibilidade a ser testada, seria a reutilização das soluções nutritivas drenadas, como está sendo preconizado atualmente em outros países (Cañadas, 1999; Amor et al., 2001; Cerda, 2001).

A falta de sincronia entre a resposta da massa seca e da produtividade de frutos ilustra a importância do fluxo hídrico no peso do produto comercial, o qual incide sobre o resultado econômico da produção. Evidencia ainda o efeito negativo da salinidade do meio radicular sobre a absorção de água pelas plantas, diminuindo ou anulando o efeito dos nutrientes sobre o crescimento da massa seca dos frutos, como demonstrado por Stanghellini et al. (1998). A regressão ajustada entre a quantidade de $\mathrm{N}$ fornecida, tomada como referencial da solução nutritiva, e a produtividade de frutos, indica a dose semanal de 101,5 mmol N/L, com a devida proporção entre os demais nutrientes, como aquela a empregar para maximizar o rendimento de frutos dessa cultura cultivada em substratos.

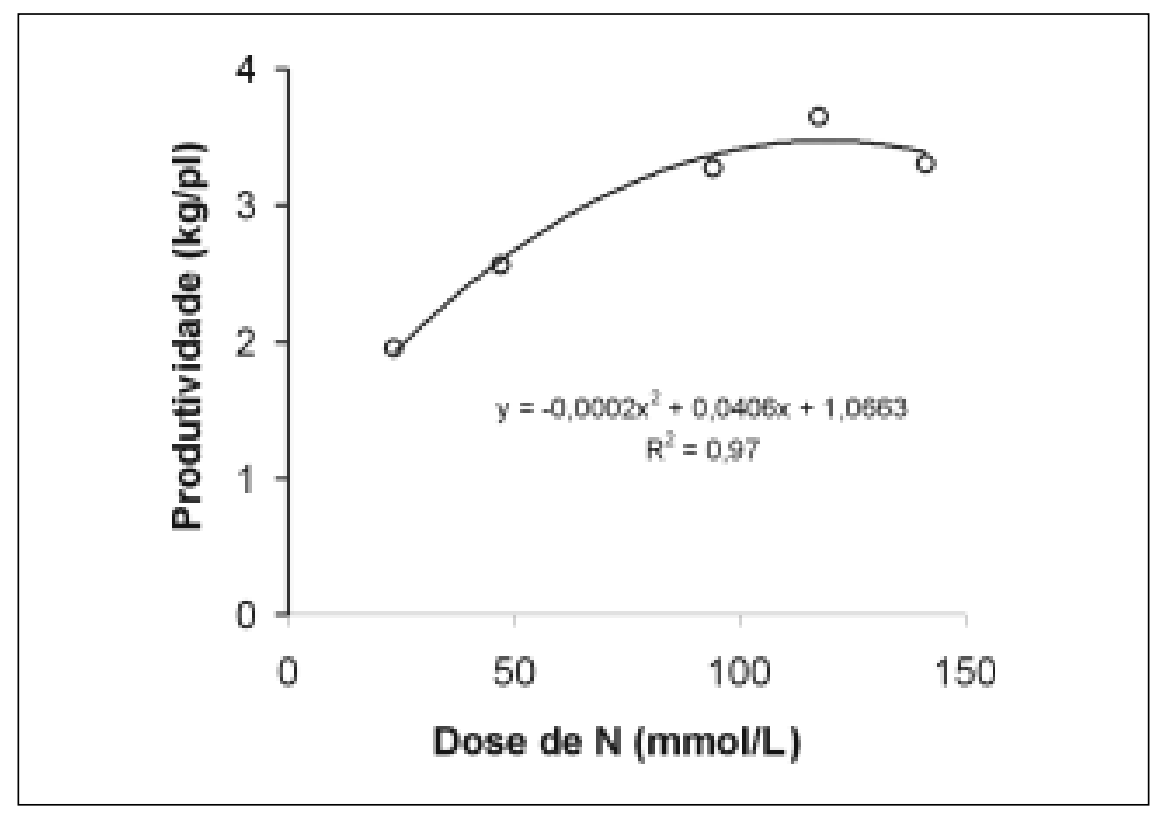

Figura 3. Curva de resposta da produção de frutos do tomateiro às quantidades de nutrientes fornecidas pelas cinco doses de solução nutritiva, empregando-se o $\mathrm{N}$ como referência. Santa Maria, UFSM, 2000.

\section{AGRADECIMENTOS}

Os autores agradecem a FAPERGS, pela concessão de uma Bolsa de Iniciação Científica ao acadêmico de Agronomia Márcio Witter. Jerônimo Luiz Andriolo é bolsista de Produtividade em Pesquisa do CNPq.

\section{LITERATURA CITADA}

ADAMS, P.; SERRA, G.; TOGNONI, F.; LEONI, S. Nutrition of greenhouse vegetables in NFT and hydroponic systems. Acta-Horticulturae, v. 361, p. 245-257, 1994.

AMOR, F.M.1; FLORES, P.; CARVAJAL, M.; MARTÍNEZ, J.M.; NAVARRO, J.M.; CERDA, A. Yield responses of soilless melon and tomato to different irrigation water qualities. Acta Horticulturae, n. 559, p. 333-338, 2001.

ANDRIOLO, J.L.; DUARTE, T.S.; LUDKE, L.; SKREBSKY, E.C. Crescimento e desenvolvimento do tomateiro cultivado em substrato com fertirrigação. Horticultura Brasileira, Brasília, v. 15, n. 1, p. 28-32, 1997.

ANDRIOLO, J.L.; POERSCKE. Cultivo de tomateiro em substratos. Santa Maria: CCR, UFSM. 1997. 13 p. (Informe Técnico, 2).

ANDRIOLO, J.L. Fisiologia das culturas protegidas. Santa Maria, Editora da UFSM, 1999. 142 p. BERTIN, N. Environnement climatique, compétition pour les assimilats et modélisation de la nouaison de la tomate en culture sous serre. Paris: Institut National Agronomique ParisGrignon, 1993, 120 p. (Thése doctorat).
CAÑADAS, J.J.M. Sistemas de cultivo en substrato: a solución perdida y com recirculación del lixiviado. In: MILAGROS, M.F.; GÓMEZ, I.M.C. (ed.). Cultivos sin suelo II. Curso Superior de Especialización. Almería: DGIFA-FIAPACaja Rural de Almería. 1999, 2ed. p. 173-205.

CORTÉS, E.M. Características del riego en cultivos sin suelo: exigencias en aportación y manejo. Resultados experimentales en cultivo de pepino en perlita. In: FERNANDÉZ, M.F.; GÓMEZ, I.M.C. ed. Cultivos sin suelo II. Almeria: DGIFA/ FIAPA/Caja Rural de Almeria. 1999. p. 287-305. CTIFL. Centre Technique Interprofessionel des Fruits e des Légumes. Maîtrise de la conduite climatique. Paris: CTIFL, 1995. 127 p.

EHRET, D.L.; HO, L.C. The effects of salinity on dry matter partitioning and fruit growth in tomatoes grown in nutrient film culture. Journal of Horticultural Science, v. 61, p. 361-367, 1986. FAYAD, J.A. Absorção de nutrientes, crescimento e produção do tomateiro cultivado em condições de campo e de estufa. Viçosa, MG: UFV, 1998. 81 p. (Dissertação mestrado).

FONTES, P.C.R.; GUIMARÃES, T.C. Manejo dos fertilizantes nas culturas de hortaliças cultivadas no solo, em cultivo protegido. Informe Agropecuário, Belo Horizonte, v. 20, n. 200/201, p. 36-44, 1999.

GRAVE, R.A. Efeito de diferentes níveis de fertilização nitrogenada sobre o crescimento e acumulação de nitrogênio na planta de tomateiro cultivado em substrato. Santa Maria: UFSM, 1999. 51 p. (Dissertação mestrado).

HUETT, D.O. Effect of nitrogen on the yield and quality of vegetables. Acta Horticulturae, n. 247, p. 205-209, 1989.

LAROUCHE, R.; GOSSELIN, A.; VEZINA, L.P. Nitrogen concentration and photosynthetic photon flux in greenhouse tomato production: I. growth and development. Journal of the American Society for Horticultural Science, v. 114, n. 3, p. 458-461, 1989. 
LE BOT, J.; ADAMOWICZ, S.; ROBIN, P. Modelling plant nutrition of horitultural crops. Scientia Horticulturae, v. 74, p. 47-82, 1998 LE BOT, J.; JEANNEQUIN, B.; FABRE, R Growth and nitrogen status of soilless tomato plants following nitrate withdrawal from the nutrient solution. Annals of Botany, v. 88, p. 361370, 2001.

MAAS, E.V. Salt tolerance of plants. Applied Agriculture Research, v. 1, n. 1, p. 12-26, 1986. PAPADOPOULOS, I. Fertilizers in irrigated agriculture and potencial pollution impact. In WATER MANAGEMENT, SALINITY AND POLLUTION CONTROL TOWARDS SUSTAINABLE IRRIGATION IN THE MEDITERRANEAN REGION". 1997, Bary, Italy. Proceedings...Bary: CIHEAM, 1997. p. 193-215.
PEREZ, M.L.S., LOPEZ, C.C. Fertirrigación de cultivos hortícolas. In: LOPEZ, C.C. ed. Fertirrigación: cultivos hortícolas y ornamentales. Madrid: Mundi-Prensa. 1998. p. 345-415.

STANGHELLINI, C.; VAN MEURS, W.T.M.; CORVER, F.; VAN DULLEMEN, E.; SIMONSE, L.; MUNIZ-CARPENA, R. Combined effect of climate and concentration of the nutrient solution on a greenhouse tomato crop. II: Yield quantity and quality. Acta Horticulturae, n. 458, p. 231237, 1998.

TAKAHASI, H.W. Nutrição e adubação do tomateiro estaqueado. In: Nutrição e adubação de hortaliças. Piracicaba: Potafos, 1993. 487 p.
VAN KEULEN, H.; STOL, W. Quantitative aspects of nitrogen nutrition in crops. Fertilizer Research, v. 27, p. 151-160, 1991.

VAN RAIJ, B. Fertilidade do solo e adubação. São Paulo: Ceres/Associação Brasileira para Pesquisa da Potassa e do Fosfato, 1991. 343 p.

VEDUIM, J.V.R.; BARTZ, H.R. Fertilidade do solo e rendimento do tomateiro em estufa de plástico. Ciência Rural, v. 28, n. 28, p. 229-233, 1998. 